\title{
Gas-Phase Anionic Complexes of Alkali Metal Ions and Peptides: Structure and Collision Activated Decompositions
}

\author{
Peifeng $\mathrm{Hu}$ and Michael L. Gross \\ Midwest Center for Mass Spectrometry, Department of Chemistry, University of Nebraska-Lincoln, Lincoln, \\ Nebraska, USA
}

\begin{abstract}
Alkali metal ions and anionic peptides can be desorbed into the gas phase to give metal-bound peptides and bis(peptide) complexes bearing a - 1 charge. Although amide nitrogens of peptide bonds are deprotonated in the gas phase by alkali metal ions, this reaction does not occur in solution. Metal-bound dipeptide anions exist as a single structure, whereas those of tripeptide complexes have three structures as revealed by tandem mass spectrometric studies. Ions of bis(peptide) complexes of alkali metals decompose upon collisional activation principally to form deprotonated peptides, in contrast to bis(peptide) complexes of alkaline earth metal ions, which undergo elimination of a neutral peptide. (] Am Soc Mass Spectrom 1994, 5, 137-143)
\end{abstract}

A lkali metal-cationized peptides can be introduced into the gas phase as (peptide + Met) ${ }^{+}$ by fast-atom bombardment (FAB), and they have been studied by tandem mass spectrometry (MS/MS) [1]. A unique decomposition reaction, the loss of the C-terminal amino acid residue of these positively charged species, has been interpreted differently with respect to the metal binding site. Some evidence was presented in favor of a structure in which the metal ion binds to the C-terminal carboxylate $[1 \mathrm{a}, \mathrm{c}, \mathrm{d}]$. Other results were interpreted to support structures in which the metal ion binds to carbonyl oxygens and other neutral functional groups $\left[1 e^{-} \mathbf{h}, 2\right]$.

Theoretical studies [2] of lithiated triglycine by MM2 calculations suggest the precursor-ion population is a distribution of structural isomers; structures in which the lithium ion is coordinated to the internal carbonyls of the neutral peptide are favored over those in which the metal binds to the C-terminus of the zwitterion [1i]. More recent work by Jensen [3], however, shows that "the semiempirical MNDO method is of insufficient accuracy for being useful." A particular point made by the author is that "the stability of the carboxylate salt is severely underestimated," but it is still less stable than structures in which $\mathrm{Li}^{+}$is bound to carbonyls. The results of MP2/6-31G*//3-21G calculations show that the structure in which the metal ion bonds at the C-terminus is one of the two most stable and is only $3 \mathrm{kcal} \mathrm{mol}^{-1}$ less stable than the most stable one [4].

Address reprint requests to Prof. Michael L. Gross, Midwest Center for Mass Spectrometry, Department of Chemistry, University of $\mathrm{Ne}$ braska-Lincoln, Lincoin, NE 68588-0304.
Unlike transition metal ions, alkali and alkalineearth metal ions do not react with peptides in solution to form anionic complexes in which amide nitrogens of peptide bonds are deprotonated [5]. We [6a, 7], as well as Adams and co-workers [6b], however, successfully generated alkaline-earth-metal-bound peptides and bis(peptide) complexes in the gas phase by using FAB and characterized their structures by MS/MS. Further, we [8] compared the metal-bonding structures with those formed by transition metal ions. The complexes of different metal groups undergo specific fragmentations that can be justified by the ligand affinities of the metals. Metal-ion ligand affinities as well as ligand acidities [9] contribute to shape the structures of the complexes.

Here we report that negative ions of alkali-metalbound peptides and bis(peptide) complexes can also be formed in the gas phase by FAB. The formation of these ions requires alkaline matrices, typically hydroxide in glycerol. This requirement is analogous to that for desorption of alkaline-earth-metal-bound peptides and bis(peptide) complexes [6]. Important clues for the structures of alkali-metal-bound peptides and bis(peptide) complexes are obtained by the characteristic fragmentations that occur upon collisional activation.

Although singly charged ions are most easily desorbed by FAB, it is possible to control the charge of the peptide by choice of the charge state of the metal ion or by selecting the charge of the desorbed complex. The peptides in anionic-peptide complexes of divalent metal ions are triply deprotonated, whereas those in the bis(peptide) complexes of divalent metal ions have a combination of -1 and -2 charge states. The pep- 
tides studied here have a charge state of either -2 (in alkali metal-bound peptides) or -1 each [in the alkali metal-bis(peptide) complexes]. Singly deprotonated peptides also exist in the positive species (peptide + Met $-\mathrm{H})^{+}$of divalent metal ions. The charge states of the peptides influence the decomposition of the complexes. Therefore, for purposes of observing intrinsic metal/peptide interactions, it is expected that the properties of the anionic complexes studied here will complement those derived from cationic alkali/peptide adducts in which the peptide exists in a neutral form.

Our interests in this subject are twofold. First, a long-range goal is to understand the gas-phase interaction of metal ions and peptides so that a solid foundation for the analogous interactions in solutions and in biological milieu can be established [1c, d]. Second, the fragmentation chemistry of these metal-peptide complexes may offer sequencing information that complements the results from protonated or deprotonated peptides. We have chosen to focus on simple, nonacidic and nonbasic peptides in the early stages so that a firm basis can be prepared for studies of more complex peptides. Because $\mathrm{FAB}$ is used to prepare the gas-phase complex, we are constrained to study singly charged metal/peptide complexes although multiply charged species may be accessible via electrospray ionization.

\section{Results and Discussion}

\section{Alkali-Metal-Bound Dipeptides}

To form an anionic complex with alkali metal ions, a dipeptide needs to be doubly deprotonated. Among the three possible deprotonation sites, the C-terminal carboxylic acid group and the amide $\mathrm{NH}$ are similarly acidic in the gas phase and have much lower $p K_{u} s$ than those of amine groups [10], such as that at the $\mathrm{N}$-terminus. We suggest that the most probable structure for alkali-metal-bound dipeptides is, therefore, one in which the metal is strongly bound both to the C-terminal carboxylate, formed by loss of $\mathrm{H}^{+}$, and the deprotonated amide nitrogen (structure 1).

The collision-activated decomposition (CAD) spectra (see Figure 1 for an example) of alkali-metal-bound dipeptides are in accord with structure 1. Major product ions include $y_{1}$ and $x_{1}$, formed by losing parts of the $\mathrm{N}$-terminal amino acid residue, as well as ions formed by losing $\mathrm{NH}_{3}$ and $\mathrm{H}_{2}$ molecules. (The convention used to denote fragment ions is the same as that proposed by Roepstorff and Fohlman [11a] and

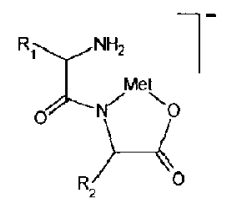

Structure 1

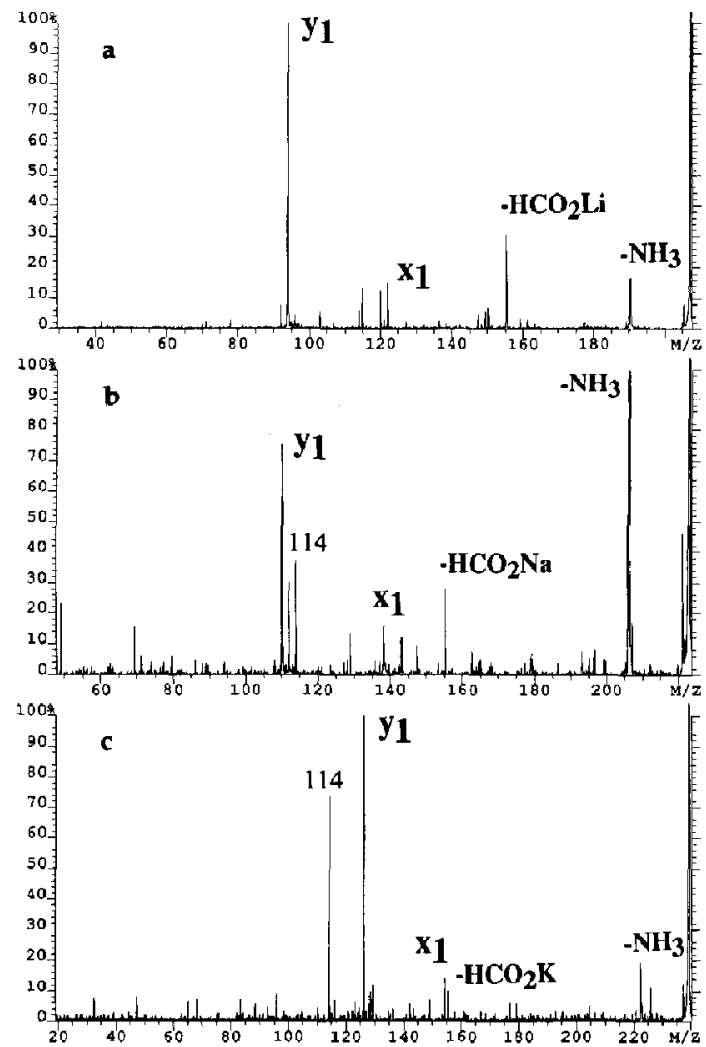

Figure 1. The CAD spectra of (a) $\mathrm{Li}^{+}-$, (b) $\mathrm{Na}^{+}$, and (c) $\mathrm{K}^{+}$ bound Leu-Ala desorbed as [pept. $\left.+\mathrm{Met}^{+}-2 \mathrm{H}^{+}\right]^{-}$.

modified by Biemann and Martin [11b]. Small letters denote the fragmentation sites, and subscript numbers specify the number of amino acid residues in the ion. The only change is that, for the purpose of maintaining a simple nomenclature, the [pept $+\operatorname{Met}^{\mathrm{n}+}-(\mathrm{n}+$ 1) $\left.\mathrm{H}^{+}\right]^{-}$ions are considered to be analogous to $(\mathrm{M}+$ $\mathrm{H})^{+}$ions. If the number of hydrogen transfers required for the formation of the metal-bound fragment is the same as that required for a protonated peptide, the nomenclature for a protonated peptide is maintained.) Formation of $y_{1}$ ions may occur by a similar mechanism to that for $y$ ions from peptide complexes involving divalent metal ions [6a, 8]. An amino hydrogen is transferred to the deprotonated amide nitrogen, leading to the losses of an imine and a $\mathrm{CO}$ molecule (Scheme I). It is noteworthy that positively charged alkali metal/peptide complexes undergo a loss of the C-terminal-amino-acid residue, whereas negatively charged alkali metal complexes undergo loss of the $\mathrm{N}$-terminal-amino-acid residue.

For those peptides that were studied here and that have a nonglycine amino acid at the $\mathrm{C}$-terminus, the ion produced by losing $\mathrm{HCO}_{2}$ Met is significant (Figure 1). For those with glycine at the C-terminus (for example, $\mathrm{Li}^{+}$-bound LG), no detectable loss of $\mathrm{HCO}_{2} \mathrm{Met}$ 


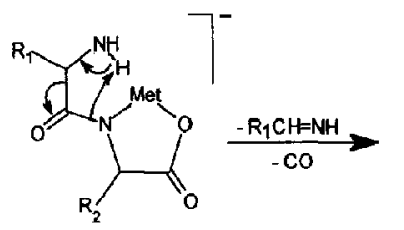

Scheme I

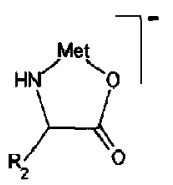

$\mathrm{y}_{1}$

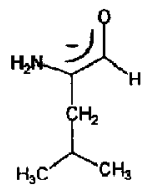

Structure 2 occurs. Apparently, for the loss to occur, the C-terminal amino acid residue must have a side chain. This implies transfer of a hydride ion from the $\beta$-carbon to the $C$-terminal carbonyl carbon and leads to the loss of $\mathrm{HCO}_{2}$ Met (Scheme II).

Other ions that do not contain the metal ion are also formed; an example is the ion of $m / z 114$ from the decomposition of alkali metal-LA complexes, as shown in the spectra of Figure $1 a-c$. The fragmentation site is also likely to be the amide group, as for the formation of $y_{1}$ ion. The charge, however, is retained on the N-terminal peptide fragment. A likely structure is 2, but the mechanism for its formation is not yet established.

\section{Alkali-Metal-Bound Tripeptides}

Because tripeptides have three sites of nearly equal acidity [10] (C-terminal $\mathrm{COOH}$ and two amide $\mathrm{NH}$ groups), deprotonation of any two of these sites results in three different structures. One involves two deprotonated amide nitrogens (3), and the other two involve the C-terminal carboxylate formed by loss of $\mathrm{H}^{+}$and one deprotonated amide nitrogen (4 and 5).

Anionic complexes of some tripeptides comprised of aliphatic amino acids undergo a facile loss of water (Figure 2). The $y_{2}$ ion is also prominent. New ions (here designated as $\mathrm{Ct}$ and $\mathrm{Nt}$ to indicate that portion of the peptide fragment bearing the charge) are formed by losing the metal and parts of the peptide (Schemes III and IV). To form the Ct ion, the metal is lost, along with the N-terminal part of the peplides. Because this ion is not formed from the anionic complex with LGP, its precursor should have structure 4 . For $\mathrm{Nt}$-ion formation, however, the metal ion is lost along with the C-terminal amino acid. The anionic complex with LGP undergoes this process, supporting the mechanism proposed in Scheme IV.

Because prolyl moieties have no amide hydrogens, anionic complexes of GPA and LGP are likely to exist as two structures (4 and 5, respectively) that involve the C-terminus in the metal bonding. The inability of these complexes to lose water upon collisional activation (Figure 3) suggests that the water elimination, when it does occur, involves a structure in which the metal binds to the two deprotonated amide nitrogens, leaving the C-terminus in a neutral form (3). The $\mathrm{Nt}$ and $y_{2}$ ions can be formed from both 4 and 5 , whereas the $\mathrm{C}_{\mathrm{t}}$ ion is probably formed from 4 (compare Figures 2 and 3). If structure 3 decomposes principally via water loss, and 3,4 , and 5 all contribute to $y_{2}$, then the contribution of structure 3 to the population of decomposing ions can be estimated by the relative abundance of the water-loss product, whereas the relative population of the decomposing structures with the C-terminus bound to the metal ( 4 and 5 ) is estimated by the total abundance of $\mathrm{Ct}$ and $\mathrm{Nt}$ ions. On this basis, the structure with a neutral $\mathrm{C}$-terminus has a higher abundance than those having a deprotonated C-terminus.

This interpretation, however, does not apply to the CAD of Li-bound GFA. We reported previously [7] that phenylalanine side chains in alkaline earth metalbis(peptide) complexes facilitate formation of a $\left(z_{n}-\right.$ $H$ ) negative ion, a product ion that competes with the $w$ ion. The Li-bound GPA anionic complex decomposes primarily to give a $\left(z_{2}-H\right)$ negative ion, and the abundance of the ion formed by losing water is approximately $30 \%$. The $\mathrm{Ct}, \mathrm{Nt}$, and $\mathrm{y}$ ions are not detectable (Figure 4). The formation of $\left(\mathrm{z}_{2}-\mathrm{H}\right)$ ion requires a decomposing structure in which the metal is bound to both the C-terminal carboxylate and the nitrogen on the amide adjoining the C-terminus (structure 4). (The production of $z$ ions is usually accompanied by that of $(z-H)$ and sometimes of $(z+H)$ product ions.)

The above evidence implicates two possibilities. First, the structures in the romposite population are separated by low barriers and interconvert before frag-

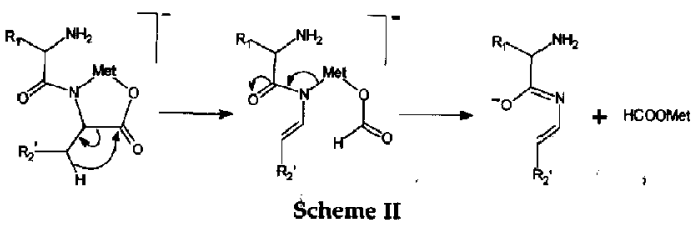

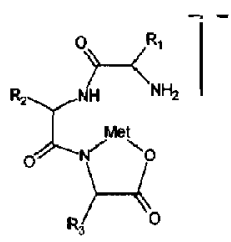

Structures $3,4,5$

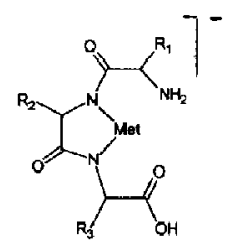

Stmethes $3,4,5$

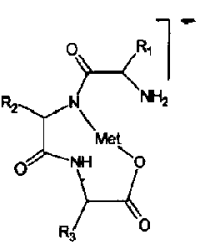




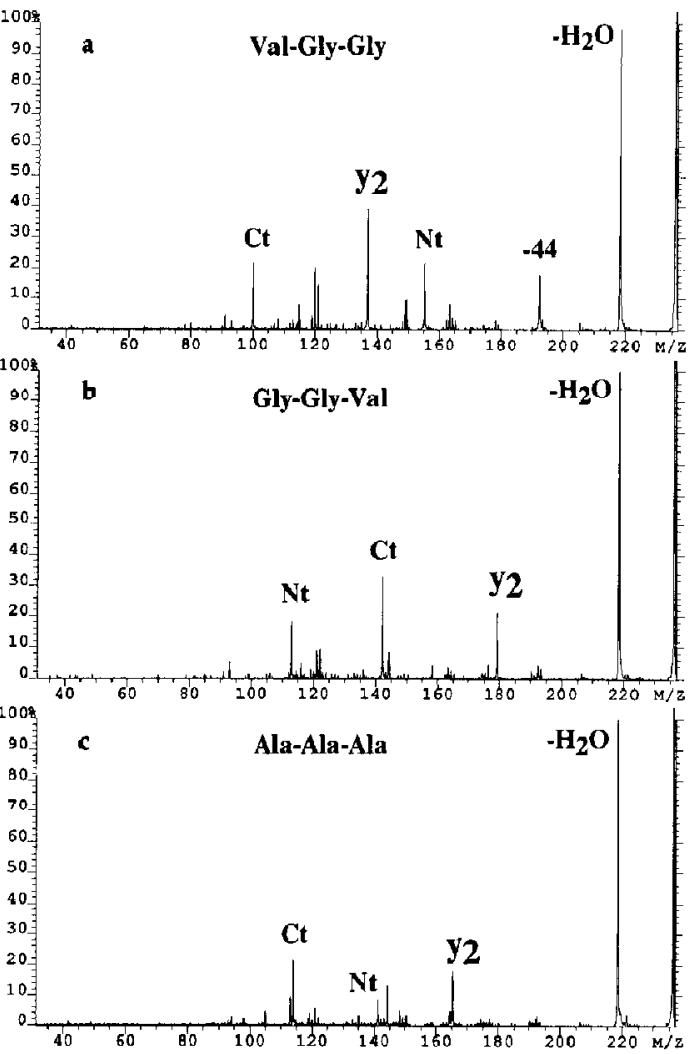

Figure 2. The $\mathrm{CAD}$ spectra of $\mathrm{Li}^{+}$-bound (a) Val-Gly-Cly, (b) Gly-Gly-Val, and (c) Ala-Ala-Ala desorbed as [pept, + Met ${ }^{+}-$ $\left.2 \mathrm{H}^{+}\right]$.

mentation occurs. For some tripeptides with aliphatic side chains, the water loss is the process of lowest energy and, thus, dominates. For the GFA anionic complex, a new, low-energy channel opens; structures 3 and 5 isomerize to 4 , and decompose to give a $\left(z_{2}-H\right)$ ion. Second, the precursors of different structures do not interconvert owing to a high energy barrier, and the relative abundances of the product

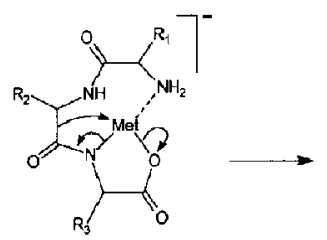<smiles>[R]C1[14CH2]NC([R8])C(=O)N1</smiles><smiles>[R9]C1C(=O)OC(=O)N1C</smiles>

Scheme III

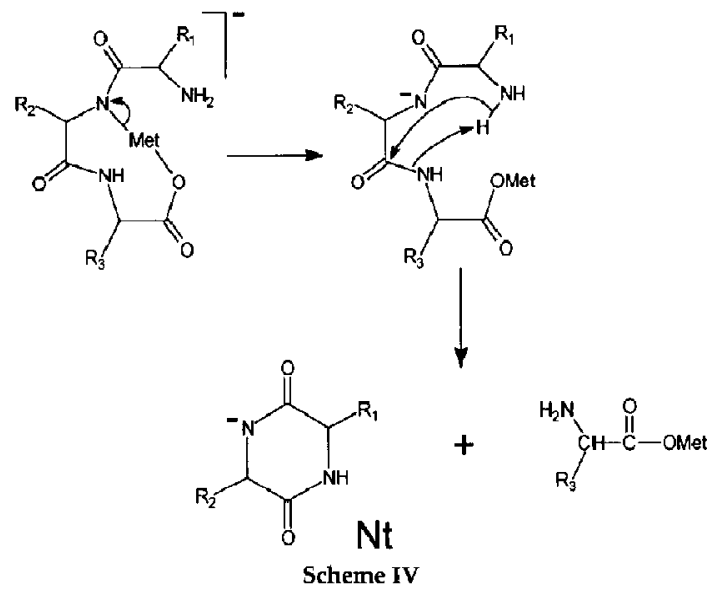

ions represent the relative population of various precursor structures. For the GFA complex, the two prominent fragmentations both occur as metastable fragmentation. "Heating" the precursors by submitting the ion beam to high-energy collisions in a cell located outside the ion source (see Experimental section) does not change the relative abundance of the two product ions, which is in accord with either a small barrier and rapidly interconverting ions or significant barriers larger than those for fragmentation. Intermediate barriers can be ruled out. Results from recent theoretical calculations $[3,4]$ suggest that the energies between various structural forms are small and, thus, the first possibility is more likely.

Tetrapeptides have a total of four sites that can be deprotonated (three amide nitrogens and the $\mathrm{C}$ terminus); they all have nearly equal gas-phase acidities [10]. Deprotonation of any two of these four sites leads to six structures. The CAD spectrum of Li-bound VAAF negative ions shows abundant products formed by losses of 18 and $44 \mathfrak{u}$, suggesting that at least for some structures there is no metal bonding to the $\mathrm{C}$ -

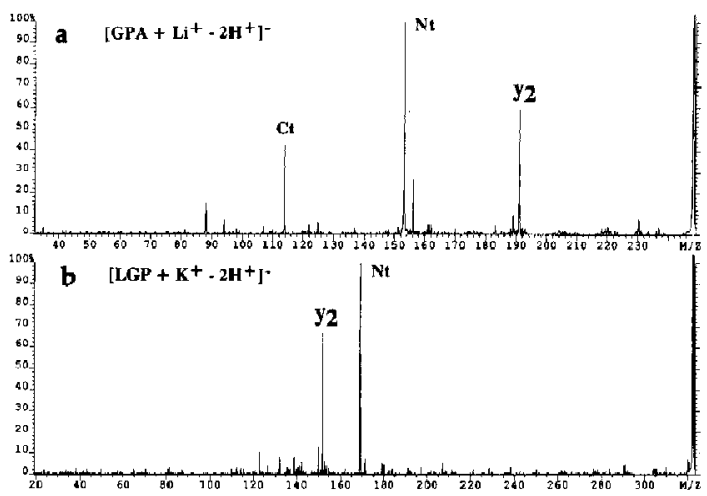

Figure 3. The CAD spectra of (a) $\mathrm{Li}^{+}$-bound GPA and (b) $\mathrm{K}^{+}$-bound Leu-Gly-Pro desorbed as [pept. $\left.+\mathrm{Met}^{+}-2 \mathrm{H}^{+}\right]^{-}$. 


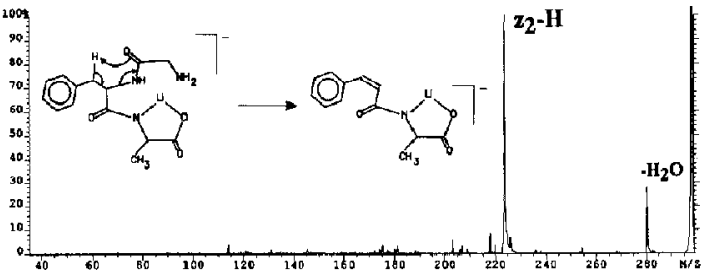

Figure 4. The CAD spectrum of $\mathrm{Li}^{+}$-bound Gly-Phe-Ala desorbed as [pept. $\left.+\mathrm{Met}^{+}-2 \mathrm{H}^{+}\right]^{-}$.

terminal carboxylate. An abundant $C_{2}$ ion (containing Li) offers support that the metal is bound at the $\mathrm{N}$ terminus, probably to the two amide functions adjoining the N-terminus. An abundant $z_{3}$ ion (as well as $z_{3}-H$ ), however, points to bonding at and near the C-terminus. These observations are in accord with a rapidly interconverting population of ion structures.

\section{Alkali Metal-Bis(Peptide) Complexes of Dipeptides / Tripeptides}

Alkali metal ions complex with two peptides to form anionic metal-bis(peptide) complexes. Upon FAB, metal-bound peptides and bis(peptide) complex anions are desorbed as minor species with respect to deprotonated peptides and matrix ions. This is in contrast to the desorption of negative ion complexes of divalent metal ions, for which the anionic complexes are major ions $[7,8]$.

The collision-induced fragmentation of alkali metalbis(peptide) complex anions yields, principally, deprotonated peptides (Figure 5). For all dipeptides and metal ions studied here, metal-bound peptide ions are minor products, and peptide-chain fragments are negligible. For $\mathrm{Li}^{+}$-bound tripeptides, deprotonated peptides are less abundant than are metal-bound peptide anions. The abundance of a metal-bound dipeptide/ tripeptide anion decreases as the metal ion size increases (abundance order is $\mathrm{Li}>\mathrm{Na}>\mathrm{K}$ ).

We reported previously $[7,8]$ that divalent, metal ion-bis(peptide) anionic complexes lose a neutral peptide to give metal-bound peptide anions. Deprotonated peptides are either of small abundances or not formed at all. For comparison, the CAD spectra of $\mathrm{Ni}^{2+}$ - and $\mathrm{Sr}^{2+}$-bis(LG) complex anions are shown in Figure 6. For complexes of the three metal ion groups (i.e., alkali, alkaline earth, or transition metal), only alkaline earth metal complex anions give facile fragmentation along the backbone of one peptide chain, forming $y_{1}$ and $z_{1}$ ions. The bis(peptide) complexes of transition and alkali metal ions show a common feature: they fragment principally by expelling one of the constituent peptides, with and without hydrogen transfer to give even- and odd-electron product ions [7, 8]. These products, however, differ in that the former usually has the metal ion as part of the ionic species,

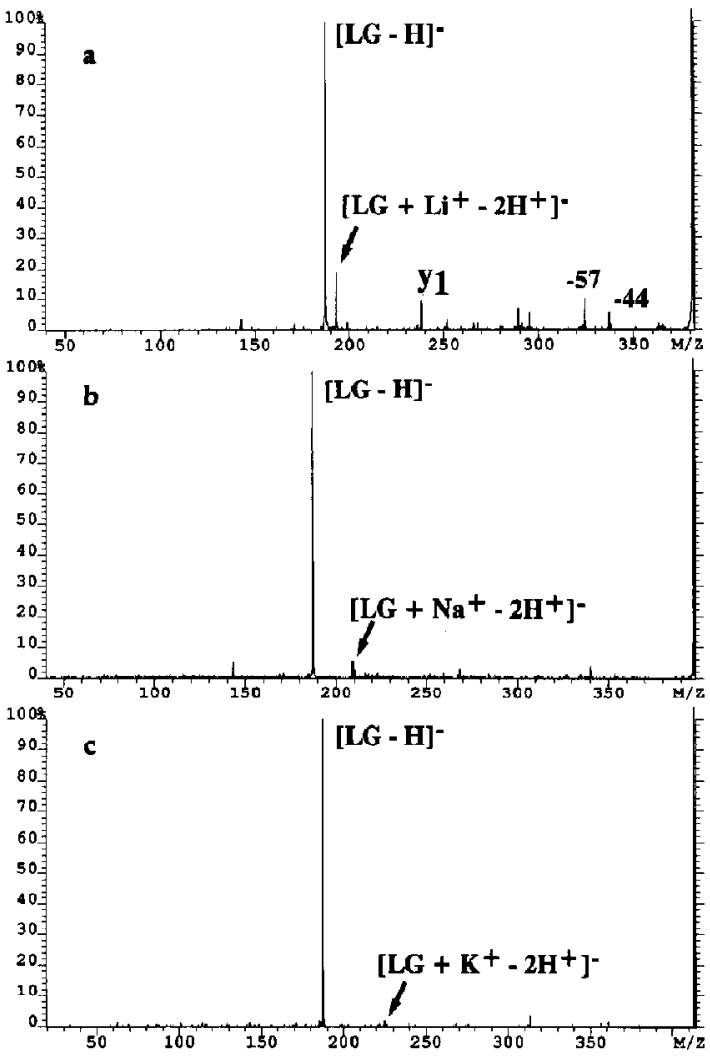

Figure 5. The CAD spectra of metal-bis(Leu-Gly) complexes of alkali metal ions: (a) $\mathrm{Li}^{+}$, (b) $\mathrm{Na}^{+}$, and (c) $\mathrm{K}^{+}$desorbed as $\left[\text { pept. }+\mathrm{Met}^{+}-2 \mathrm{H}^{+}\right]^{-}$.

whereas the alkali metal complexes have the metal ion as part of the neutral.

We have attributed the differences in the fragmentations of peptide complexes of transition metal and alkaline earth metal ions to broad differences in ligand affinity [8]. The oxyphilic property of alkaline earth

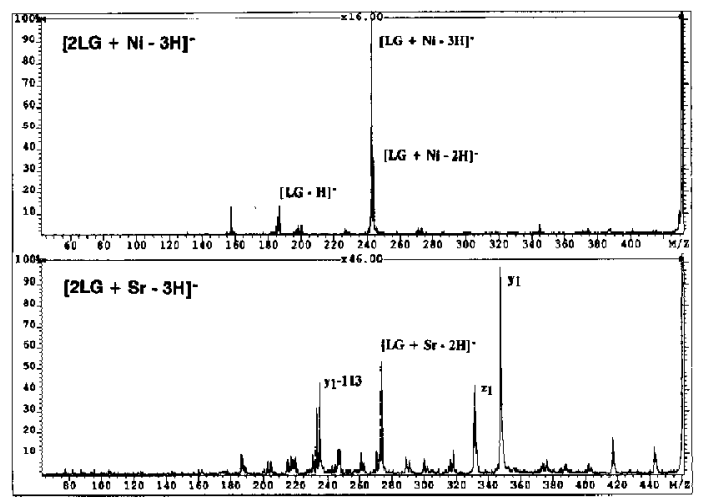

Figure 6. The CAD spectra of $\mathrm{Ni}^{2+}$-bis(Leu-Gly) complex and $\mathrm{Sr}^{2+}$-bis(Leu-Gly) complex. 
metal ions imparts strong bonding to a bis(peptide) complex because two of the three metal binding sites are oxygen ligands. The transition metal ions studied here, on the other hand, favor nitrogen ligands, and the bis(peptide) complexes decompose to metal-bound peptides that have two of the three charge sites on nitrogens. A pertinent review of condensed-phase bonding and structures is available [12].

For fragmentation of alkali metal-bis(peptide) complexes, other factors such as negative-to-positive charge ratios may play a role [12]. For complexes of doubly charged metal ions, the ratio is $3: 2$, but for complexes of singly charged metal ions the ratio is $2: 1$. A ratio of 3:2 may signal that metal-ligand charge attraction and ligand-ligand repulsion are nearly balanced. For a ratio of $2: 1$, however, the charges are less balanced, and the repulsion of the two negatively charged ligands may overwhelm the charge attraction between the metal and the ligands. This instability leads to the formation of a deprotonated peptide as the major product, and the metal ion is lost as a part of the neutral in the fragmentation.

\section{Conclusion}

Alkali metal ions can interact with peptides to form ionic, gas-phase complexes in which the peptides are doubly deprotonated. Other species such as neutral carboxylate salts may also be produced, but they are not measured in this work. The nature of the interactions in the charged complex differ from those of divalent metal ions. Anionic alkali metal-bound peptides decompose to both metal-containing and nonmetal-containing anionic fragments. For example, a metal salt of the C-terminal amino acid is lost from alkali metal complexes but not from alkaline earth complexes. Doubly charged metal ions usually remain as part of the product ions. These differences are attributed to the differences in ligand-to-metal charge ratios in the two classes of complexes.

\section{Experimental}

\section{Reagents}

The peptides used in this work were commercially available from Sigmla Chemical Co. (St. Luuis, MO). Alkaline earth metal ion hydroxides were from Fisher Scientific Company (Fair Lawn, NJ). Glycerol and thioglycerol were purchased from Aldrich Chemical Co. (Milwaukee, WI).

\section{Instrumentation}

The mass spectrometer used for acquiring the mass spectra and tandem mass spectra was a VG four-sector ZAB-T (VG Analytical, Manchester, UK). The instrument consisted of two high-mass, double-focusing mass spectrometers [13]. The design of MS-II is a reverse geometry Mattauch-Herzog type (BE). The instrument was equipped with a $\mathrm{Cs}^{+}$gun that provided a $17-\mathrm{keV}$ $\mathrm{Cs}^{+}$beam (the overall energy for desorption of negative ions was $25 \mathrm{keV}$ given that the sample probe was at $-8 \mathrm{kV}$ ). When mass spectra were acquired, only MS-I and the intermediate detector were used. When MS/MS experiments were conducted, MS-I was used to select the precursor ion at a mass resolution of approximately 1500 , and a B/E scan was taken with MS-II to record the product ions produced by collisional activation in the collision cell located between MS-I and MS-II. The object slit of MS-II was closed so that the peak of the selected ion went from flat to round top (slit fully illuminated) so that the mass resolving power for the product ions was ca. 1000 (fwhh).

\section{Procedures}

For FAB-MS/MS experiments, a few micrograms of the peptide were added to the stainless steel tip of a FAB probe and then mixed with glycerol that was saturated with alkali metal ion hydroxide. The tip was then exposed to $25-\mathrm{keV} \mathrm{Cs}{ }^{+}$beam for the desorption of the complexes of interest.

For internal energy-enhancement experiments, the Kratos MS-50 triple sector instrument (Kratos Analytical, Manchester, UK) was used [14]. The sourceproduced alkali metal/peptide complex beam was attenuated by $80 \%$ at the collision cell located between the source and the electric sector to elevate the internal energy of the precursor ions. The $\beta$-slit was closed from the high energy side to attenuate the beam by approximately another $80 \%$ so that the population of the precursors with high internal energies (those that were slowed by collision) was increased. CAD spectra were then recorded by scanning the final electrostatic analyzer (a mass-analyzed ion kinetic energy spectrometry experiment).

\section{Acknowledgments}

This work was supported by the National Science Foundation (grant no. CHE9017250). We acknowledge the constructive comments of one of the reviewers and the assistance of Dr. D. Giblin with some of the experiments.

\section{References}

1. (a) Renner, D.; Spiteller, G. Biomed. Environ. Mass Spectrom. 1988, 15, 75-77. (b) Tang, X. J.; Ens, W.; Standing, K. G.; Westmore, J. B. Anal. Chem. 1988, 60, 1791-1799. (c) Grese, R. P.; Cerny, R. L.; Gross, M. L. J. Am. Chem. Soc, 1989, 111, 2835-2842. (d) Grese, R. P.; Gross, M. L. J. Am. Chem. Soc. 1990, 112, 5098-5104. (e) Russell, D. H; McGlohon, E. S; Mallis, L. M. Anal. Chem. 1988, 60, 1818-1824. (f) Mallis, L. M.; Russell, D. H. Anal. Chem. 1986, 58, 1076-1080. (g) Teesch, L. M.; Adams, J. J. Am. Chem. Soc. 1991, 113, 812-820. (h) Teesch, L. M.; Orlando, R. C.; Adams, J. J. Am. Chem. Soc. 
1991, 113, 3668-3675. (i) Leary, J. A.; Zhou, Z. R.; Ogden, S. A.; Williams, T. D. J. Am. Soc Mass Spectrom. 1990, 1, $473-480$.

2. Leary, I. A.; Zhou, Z. R.; Ogden, S. A.; Williams, T. D. J. Am. Soc. Mass Spectrom. 1990, $1473-480$.

3. Jensen, F. J. Am. Chem. Soc. 1992, 114, 9533-9537.

4. Bouchonnet, S.; Hoppilliard, Y. Org. Mass Spectrom. 1992, 27, $71-76$.

5. Sigel, H.; Martin, R. B. Chem. Rev. 1982, 82, 385-426.

6. (a) Hu, P. F.; Gross, M. L. J. Am. Chem. Soc. 1992, 114, 9153-9160. (b) Zhao, H.; Reiter, A.; Teesch, L. M.: Adams, J. J. Am. Chem. Soc. 1993, 115, 2854-2863.

7. Hu, P. F.; Gross, M. L. J. Am. Chem. Soc. 1992, 114, 9161-9169.
8. Hu, P. F.; Gross, M. L. J. Am. Chem. Soc, 1993, 115, 8821-8828.

9. Teesch, L. M.; Adams, J. J. Am. Chem. Soc. 1990, 112, $1110-4120$.

10. (a) Bartmess, J. E. Mass Spectrom. Rev. 1989, 8, 297-343. (b) Meot-Ner (Mautner), M. J. Am. Chem. Soc. 1988, 110, 3071-3075.

11, (a) Roepstorff, P,; Fohlman, J. Biomed. Mass Spectrom. 1984, 11, 601. (b) Biemann, K.; Martin, S. A. Mass Spectrom. Rev. $1987,6,1-76$

12. Glusker, J. P. Adv. Protein Chem. 1991, 42, 1-77.

13. Gross, M. L. In Methods in Enzymology, Vol. 193. MoCloskey, J. A., Ed. Academic: New York, 1990; pp 131-153.

14. Gross, M. L.; Chess, E. K.; Lyon, P. A.; Crow, F. W.; Evans, S.; Tudge, $\mathrm{H}$. Int. J. Mass Spectront. Ion Phys. 1982, 42, 243-254. 